\title{
Formation of graphene nanostrctures on diamond nanowire surfaces
}

\author{
Susumu Okada ${ }^{a, b, *}$, \\ ${ }^{a}$ Graduate School of Pure and Applied Sciences and Center for Computational \\ Sciences, University of Tsukuba, Tsukuba, Ibaraki 305-8571, Japan \\ b Japan Science Technology Agency, CREST, Sanbancho, Chiyoda-ku, Tokyo \\ 102-0075, Japan
}

Received 18 September 2009; in final form 7 October 2009

\begin{abstract}
We carried out first-principle total-energy calculations on carbon nanowires with nanometer-scale diameters up to $4.3 \mathrm{~nm}$ consisting of $\mathrm{sp}^{3}$ carbon atoms. Due to the substantial surface reconstruction, the nanowires are energetically stable although their surfaces are not terminated by any atoms. The calculated total energy of the wire with $4.3 \mathrm{~nm}$ diameter is nearly equal to that of the single-walled nanotube with $1 \mathrm{~nm}$ diameter. The surface reconstruction results in graphitization of the outermost shell of the wire which is expected to cause the structural transformation from diamond to the multi-shell nanotube or nanoscale graphene ribbons. The electronic structures of the wires are semiconducting with a moderate indirect energy gap.
\end{abstract}

\section{Introduction}

Miniaturization in semiconductor technology is driving us to understand and to predict the physical properties of materials with nanometer scale for their possible applications in current and future technologies. Indeed, carbon nanotubes [1,2] and graphene [3] have held a premier position in the field of current nanotechnology. In nanoscale materials, it has been demonstrated that many factors are crucial for determining their physical properties: Local atomic arrangements [4], global network topology [5-7], dimensionality [8,7], surfaces [9-11], and sizes are typical factors which cooperatively or competitively affect each other, resulting in unusual properties that are not found

* Corresponding author. FAX: +81-29-853-5924

Email address: sokada@comas.frsc.tsukuba.ac.jp (Susumu Okada). 
in bulk materials. As for carbon, the tubular form of graphene [12] is either a metal or a semiconductor depending on the atomic arrangement along the circumference $[5,6]$. By contrast, when applying the open boundary condition on graphene, its nano-ribbons with zigzag edges exhibit magnetic ordering around their edges [9-11].

Besides the topological aspects, the nano-scale surfaces are highly important in covalent materials. It is well known that dangling bonds generated at surfaces are expected to play crucial roles in atomic structures of their surfaces [13]: Reconstruction of surface atoms usually reduces dangling-bond energies albeit with increasing stress energies. Such competition between electronic and elastic energies produces a rich variety of surface geometries. For $\mathrm{Si}(111)$ surfaces, the top layer Si atoms constitute a triangular lattice and the buckling of the atoms along the [110] direction leads to a $2 \times 1$ structure [14,15]. In addition to bulk surfaces, a recent calculation on the Si nano-surfaces shows that the surface spin polarization takes place due to the small flexibility for surface reconstructions.

In recent times, the physical properties of one-dimensional materials comprising of three-fold coordinate carbon atoms, e.g. carbon nanotube and graphene nano-ribbons, have been well studied. However, little is known of the onedimensional materials consisting of four-fold coordinated carbon atoms. In this case, it is expected that the interplay between electronic and elastic energies is expected to produce a rich variety of surface geometries and electronic structures of nano-scale tubular forms of the four-fold coordinate $\mathrm{C}$ atoms due to surface $\mathrm{C}$ atoms possessing unsaturated bonds. Furthermore, in this case, the competition between $\mathrm{sp}^{2}$ and $\mathrm{sp}^{3}$ hybridizations also plays a crucial role in determining their structures and electronic properties. Besides the structural viewpoint, the nano-scale rods of diamond are expected to be precursors for other nanoscale $\mathrm{C}$ materials with three-fold coordination, such as carbon nanotubes. Thus, it is interesting to investigate the energetics and electronic properties of nano-wires of $\mathrm{C}$ atoms with four-fold coordination.

The purpose of the present paper is to get a qualitative insight into the electronic properties and energetic stability of a nanowire of a hexagonal diamond with clean surfaces by calculation of total-energy electronic-structure calculations by the density functional theory (DFT). Our DFT calculations show that the diamond nanowires are energetically stable, the total energy being slightly higher than those of single-walled and multi-walled carbon nanotubes with similar diameters. Although the surfaces of the wires are unsaturated by $\mathrm{H}$ atoms, the total energy of a diamond nanowire with its diameter of $\sim 4 \mathrm{~nm}$ is nearly equal to that of the single-walled nanotube with $1 \mathrm{~nm}$ diameter. The remarkable stability of the diamond nanowires is ascribed to the graphitization of the outermost shell of the wires by transforming from the $\mathrm{sp}^{3}$ to $\mathrm{sp}^{2}$ hybridizations of the surface atoms. The graphitization also results in estab- 

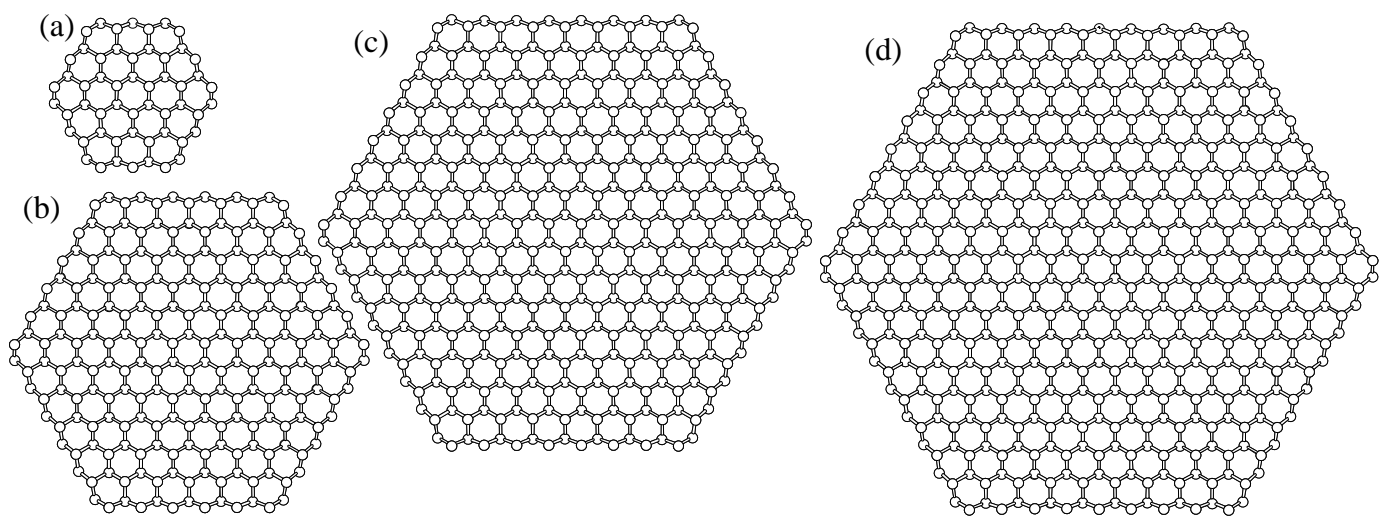

Fig. 1. Top views of the optimized geometries of diamond nanowires with diameters of (a) $d=1.26 \mathrm{~nm}$, (b) $d=2.77 \mathrm{~nm}$, (c) $d=3.78 \mathrm{~nm}$, and (d) $d=4.28 \mathrm{~nm}$.

lishment of the semiconducting electronic properties of the wires by decreasing the number of unpaired electrons associated with the dangling bonds. A further analysis has revealed that the wire can be the basis for other nano-scale graphitic materials such as graphene nano-ribbons and carbon nanotubes, by peeling off their outermost shells.

\section{Calculation methods and models}

All calculations were performed using the density functional theory (DFT) $[18,19]$. For the exchange-correlation energy among electrons, we used a functional form fitted to the Monte-Carlo results for the homogeneous electron gas [20,21]. To describe the electron-ion interaction, we use pseudopotentials with the Kleinman-Bylander scheme [22]. The valence wave functions were expanded by the plane-wave basis set with a cut-off energy of $36 \mathrm{Ry}$, which is sufficient to give qualitative discussions on the relative stability and electronic properties of carbon-related materials [23]. The conjugate-gradient minimization scheme was used both for the electronic-structure calculation and for the geometry optimization. The structural optimization was performed until the remaining forces were less than $5 \mathrm{mRy} / \AA$. We adopted a supercell model in which a diamond nanowire was placed with its surfaces being separated by at least $0.7 \mathrm{~nm}$ from other atoms of adjacent wires to simulate an isolated nanowire. Integration over a one-dimensional Brillouin zone was carried out using equidistant $k$-point sampling in which six $k$-points were taken along a wire direction. Since the number of unsaturated bonds on surfaces were crucial in this calculation, for the stability of $\mathrm{sp}^{3}$ networks [17], we considered the hexagonal diamond nanowires for which the wire axis was parallel to the (111) direction of the hexagonal diamond. To compare the energies with the carbon nanotubes, we also performed the total energy calculations on double-walled, triple-walled, and quadruple-walled nanotubes comprising of zigzag carbon nanotubes. 


\section{Results and Discussion}

\subsection{Geometric structure of nanowires}

Figure 1 shows the optimized structures of diamond nanowires with clean surfaces and diameters less than $4.5 \mathrm{~nm}$. Obviously, significant structural relaxations are found to take place in the outermost shell of the nanowires: The outermost shell is considerably flattened indicating that the atoms situated at the outermost shell are transformed into $\mathrm{sp}^{2}$ hybridization irrespective of the wire diameter. Indeed, the optimized bond length in the outermost shell ranges from 0.134 to $0.149 \mathrm{~nm}$ indicating the evidence of $\mathrm{sp}^{2}$ rather than $\mathrm{sp}^{3}$ hybridizations. In addition, significant modulation on the inter-shell bonds connecting the outermost and the inner shells is also found. The bond length of such a bond is $0.163 \mathrm{~nm}$, which is slightly elongated compared with the typical $\mathrm{sp}^{3}$ bond. The radial distribution function (RDF) of the nanowires clearly indicates these structural relaxations (Fig. 2). Around the first peak in the RDF for all wires, which corresponds to the $\mathrm{sp}^{3}$ bond length, two additional structures were found; they were attributed to the $\mathrm{sp}^{2}$-like bond in the outermost shell and the inter-shell bonds. The ratio of the outermost atoms monotonically decreases with increasing the wire diameter so that the characteristic structures decrease with increasing nanowire diameter.

The elongated inter-shell bond indicates that the outermost shell of the nanowire can be regarded as corrugated graphene loosely bound to its inner shell. Thus, as expected, the outermost shell can be peeled off from the core region of the diamond nanowire. Indeed, the calculated activation barrier for peeling of one of the six facets is found to be $0.7 \mathrm{eV}$ per bond, confirming that the outermost shell of the diamond nanowire is easily peeled from the wire under certain conditions such as thermal annealing at appropriate temperature [Fig. 3]. Moreover, our results also indicate the possibility of the structural transformation from diamond nanowires into multi-walled carbon nanotubes by successive peeling of each shell of nanowires with the supply of excess $\mathrm{C}$ atoms. A previous calculation also showed that the surfaces of the diamond nano-balls are spontaneously peeled off resulting in the graphitic structure with subnanometer size [24]. In this case, the spherical surface of the diamond nano-ball causes the pentagonal ring in their graphitic structure. In sharp contrast, on the diamond nanowire, the resultant graphitic structure sorely consists of hexagonal rings. 

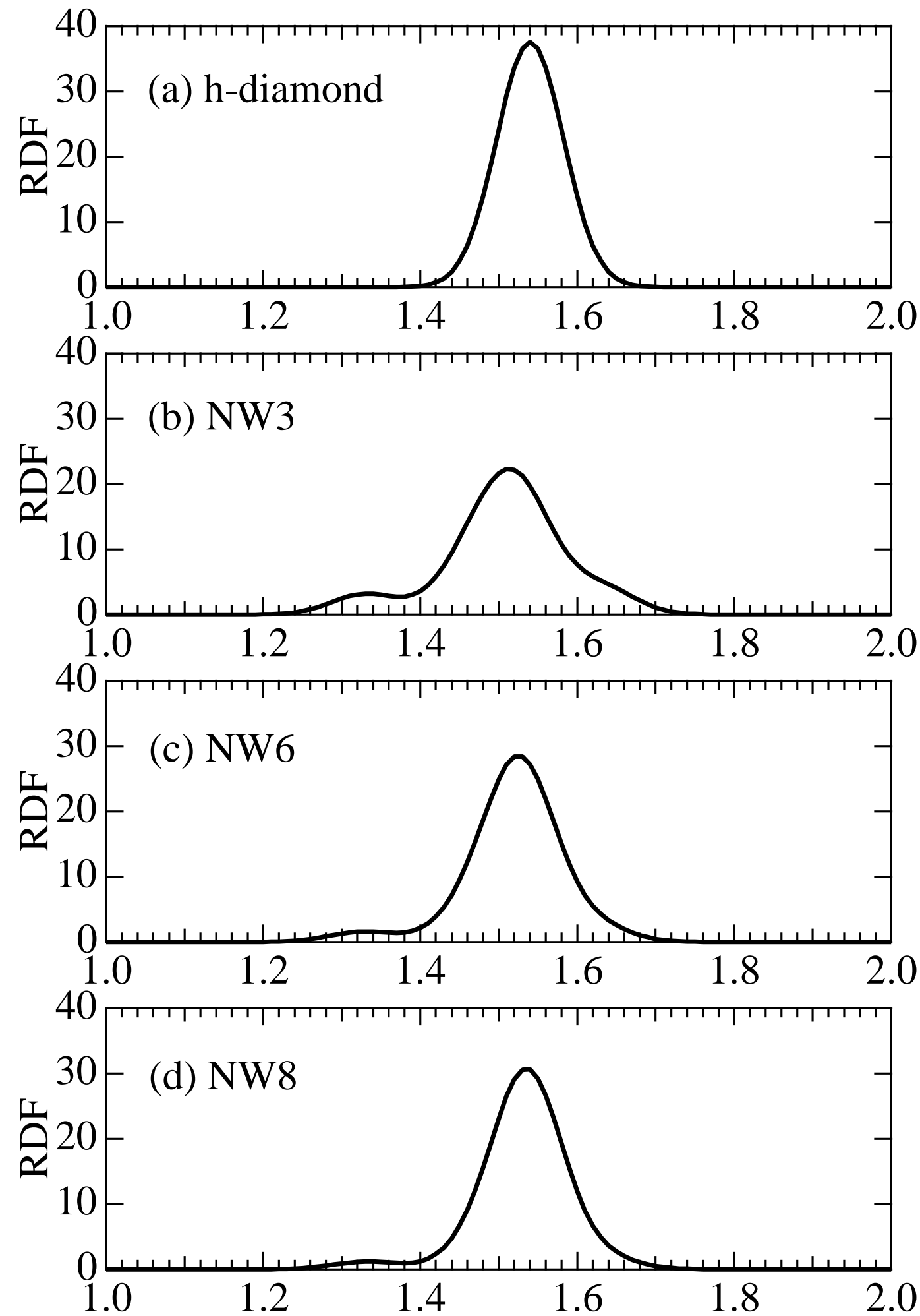

Fig. 2. Radial distribution functions around the nearest neighbor atoms of (a) hexagonal diamond (h-diamond), (b) the nanowire with diameter $d=1.26 \mathrm{~nm}$ (NW3), (c) the nanowire with diameter $d=2.77 \mathrm{~nm}(\mathrm{NW} 6)$, and (d) the nanowire with diameter $d=3.78 \mathrm{~nm}(\mathrm{NW} 8)$. 


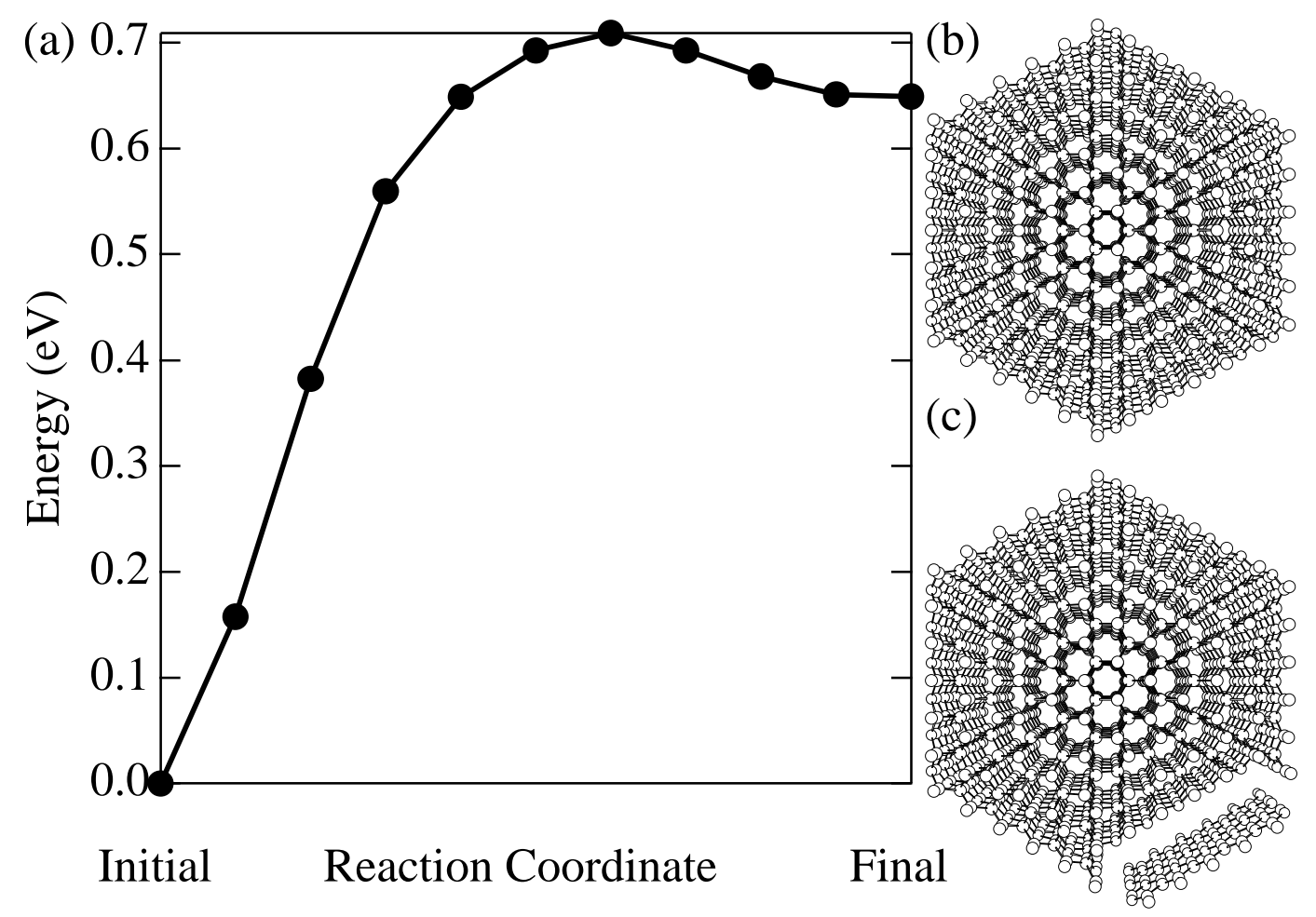

Fig. 3. (a) An activation barrier for peeling of the outermost shell of the diamond nanowire with diameter $d=3.78 \mathrm{~nm}$. Optimized atomic structures of (b) the initial and (c) the final geometries on the surface peeling.

\subsection{Energetics of nanowires}

The surface reconstruction of the diamond nanowire implies that the nanowires are energetically stable. Figure 4 shows the total energy per atom of diamond nanowires as a function of the wire diameter as well as those of the single-, double-, triple-, and quadruple-walled carbon nanotubes. As shown in Fig. 4 , the total energy of nanowires is slightly higher than that of the carbon nanotubes with similar diameters, so that the wires are an energetically stable form of the carbon allotropes. For instance, the total energy of the nanowire with $4.3 \mathrm{~nm}$ diameter is nearly equal to that of the single-walled nanotubes with $1 \mathrm{~nm}$ diameter. This remarkable stability of the nanowires is ascribed to the absence of the dangling bond on their surfaces resulting from the structural transformation from $\mathrm{sp}^{3}$ to $\mathrm{sp}^{2}$ hybridizations on the $\mathrm{C}$ atoms situated in the outermost shell. The total energy gradually approaches that of the graphene with increasing the diameter. However, the asymptotic behavior is slightly different from that of the nanotubes in which the energy is proportional to the inverse of their diameter.

The remarkable energetic stability of the nanowires may also provide a key to the formation mechanisms of single-walled carbon nanotubes. It has been demonstrated by experiments that there is a crossover between single-walled 
carbon nanotube and the multi-walled nanotube or carbon nanowires with amorphous forms with $\mathrm{sp}^{3}$ carbons: single-walled nanotubes are favorable for the catalyst nanoparticles with diameter less than $4 \mathrm{~nm}$, while the carbon nanowires are favorable for the catalyst nanoparticles with diameter longer than $4 \mathrm{~nm}$ [25]. Since the energy of a nanowire with $4 \mathrm{~nm}$ diameter is nearly equal to that of a single-walled nanotube with $1 \mathrm{~nm}$ diameter, the energy per area of the nanowire is lower that that of the single-walled nanotubes. Thus, the nanowires with large diameter are favorable structures for catalyst nanoparticles with diameters of $4 \mathrm{~nm}$ or larger. The energetics of the nanowires calculated here may explain the origin of structural crossover at $\sim$ $4 \mathrm{~nm}$ diameter for catalysts.

\subsection{Electronic structure of nanowires}

Figures 5(a), (b), and (c) show the electronic energy band structures of the nanowires with diameters of $1.26,2.77$, and $3.78 \mathrm{~nm}$, respectively. The electronic structure of the nanowires is semiconducting with an indirect energy gap irrespective of the wire diameter. The calculated fundamental energy gap is $1.95,2.13,2.28,1.99,1.86,1.73$, and $1.65 \mathrm{eV}$ for nanowires with diameters of $0.75,1.26,1.76,2.27,2.77,3.27$, and $3.78 \mathrm{~nm}$, respectively. It should be noted that there are two classifications of nanowires in terms of the detailed electronic structures around the fundamental energy gap. For thin nanowires with a diameter smaller than $1.5 \mathrm{~nm}$, the gap increases with increasing diameter. In this diameter region, the top of the valence band is located at the $\Gamma$ point, while the bottom of the conduction band is at the middle of the one-dimensional Brillouin zone. By contrast, the gap decreases with increasing diameter for nanowires with $1.5 \mathrm{~nm}$ or larger diameters. For these nanowires, although the top of the valence band is also at the $\Gamma$ point, the bottom of the conduction band is located on the Brillouin zone boundary at the $\mathrm{X}$ point.

The characteristics of the electronic structures around the fundamental gap affects the effective masses of the hole and electron at the bottom of the conduction band and the top of valence band, respectively. The calculated effective masses of the holes are 1.3, 3.1, 0.4, 0.7, 0.7, and $0.6 \mathrm{~m}_{\mathrm{e}}$ for nanowires with diameters of $0.75,1.26,1.76,2.27,2.77$, and $3.78 \mathrm{~nm}$, respectively. In contrast, the masses of the electrons are $0.9,2.1,5.5,2.6,1.0$, and $1.0 \mathrm{~m}_{\mathrm{e}}$ for nanowires with diameters of $0.75,1.26,1.76,2.27,2.77$, and $3.78 \mathrm{~nm}$, respectively. The results indicate that the effective masses of both the hole and electron are independent of the wire diameter for diameters of $3 \mathrm{~nm}$ or longer.

We now focus on the nanowire with $3.78 \mathrm{~nm}$ diameter [Fig. 5 (c)]. Due to the large number of electrons in the cell ( $\sim 3000$ electrons/cell), we could not 


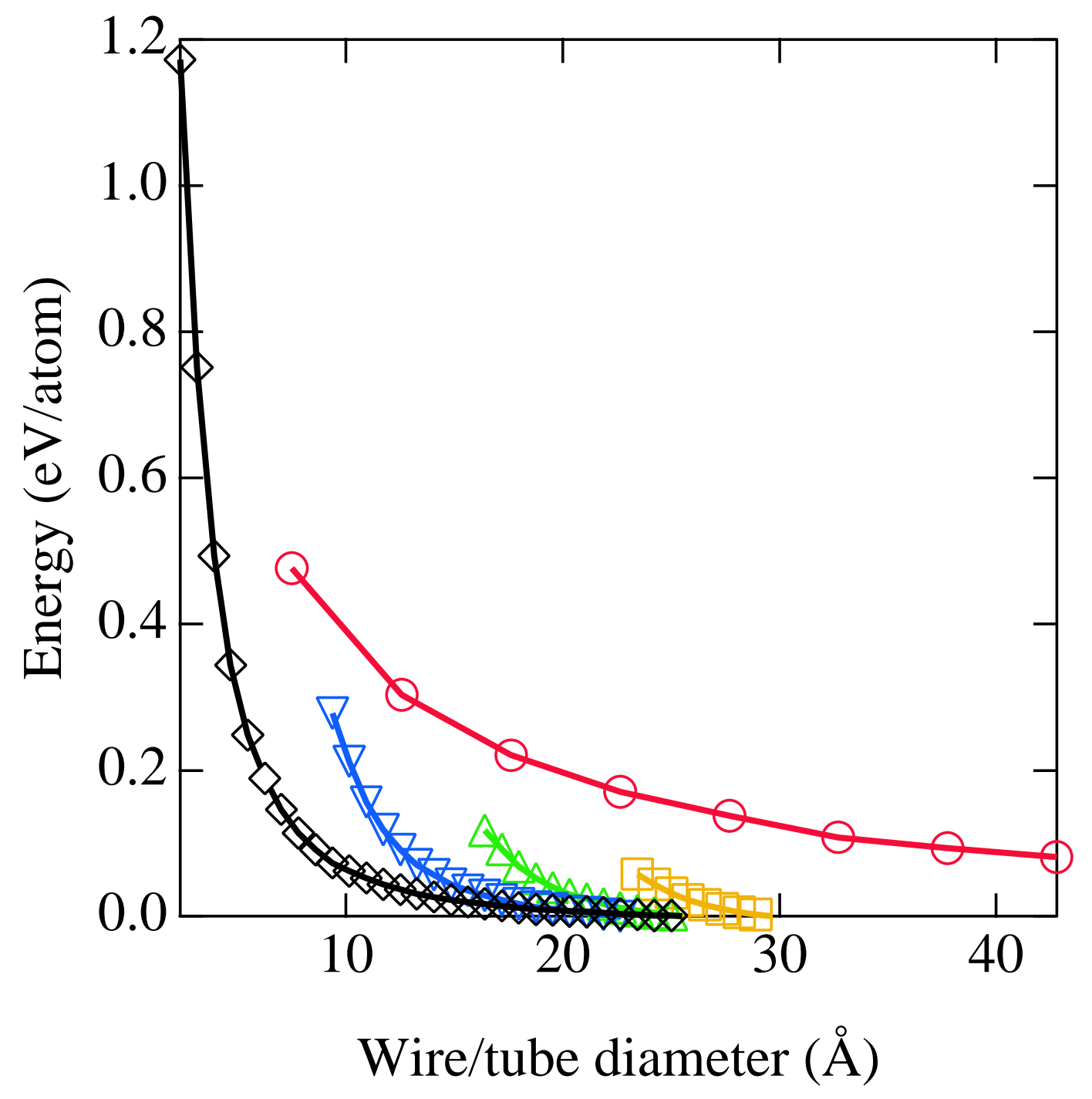

Fig. 4. (Color online) Total energy per atom of single-walled nanotubes, multi-walled nanotubes, and diamond nanowires as a function of their diameters. The total energies of diamond nanowires are denoted by red circles, quadruple-walled nanotubes by yellow squares, triple-walled nanotubes by green triangles, double-walled nanotubes by blue triangles, and single-walled nanotubes by black diamonds. Energies are measured from that of the isolated graphene sheet.

find a significant structure in the electronic energy band and found an almost continuous spectrum for the valence energy band of the nanowire. The largest energy spacing in the deep-energy region is $200 \mathrm{meV}$, while the spacing is less than $10 \mathrm{meV}$ in the shallow-energy regions. Indeed, the density of states for the valence band clearly indicates its bulk diamond character rather than a one-dimensional material. In particular, the onset of the density of states agrees closely with that of the diamond. In contrast to the valence band, the conduction band evidently possesses some structures. The lowest branches of the conduction band are found to be almost isolated from the other conduction band and possess a relatively flat dispersion. This branch has its minimum at 


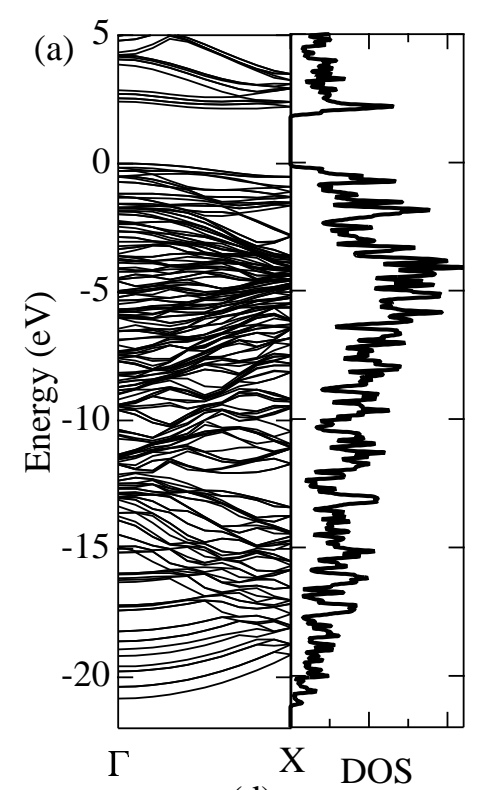

(d)

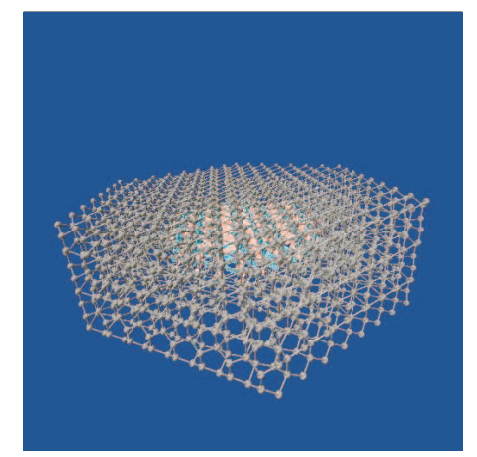

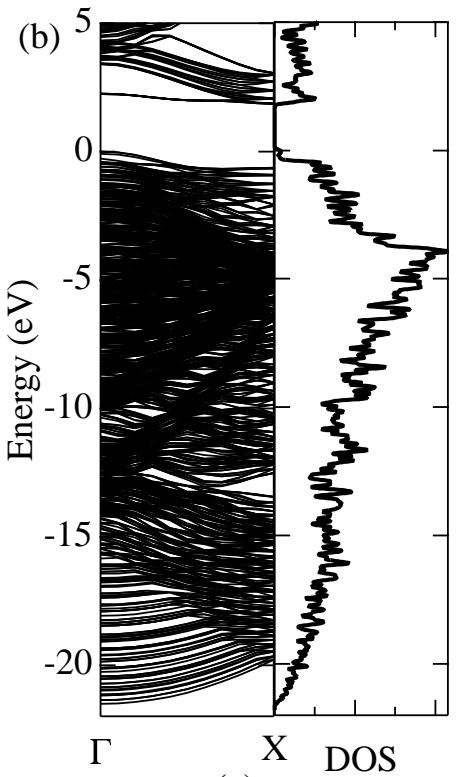

(e)

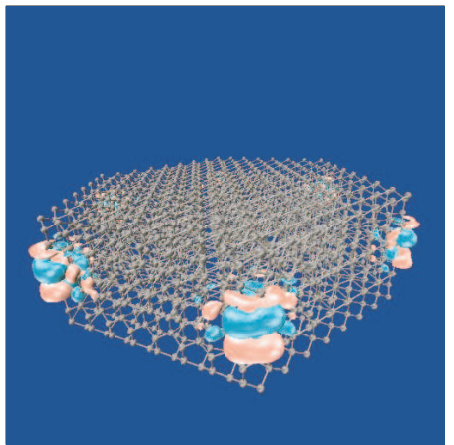

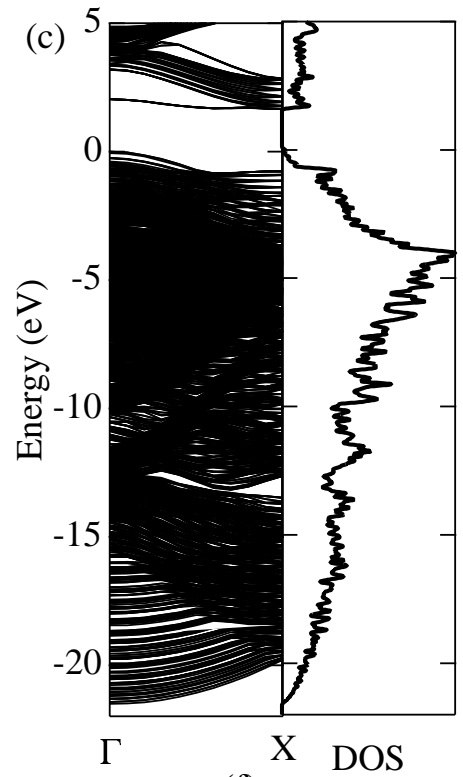

(f)

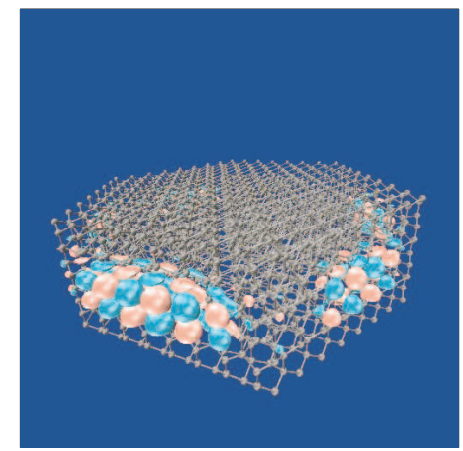

Fig. 5. (Color online) (a) Electronic structure of nanowires with (a) diameter $d=1.26$ $\mathrm{nm}$, (b) diameter $d=2.77 \mathrm{~nm}$, and (c) diameter $d=3.78 \mathrm{~nm}$. The isosurfaces of squared wave functions of the nanowire with diameter $d=3.78 \mathrm{~nm}$ at the $\Gamma$ point for (d) valence band top, (e) the lowest branch of the conduction band, and (f) the second lowest branch of the conduction band. The energies are measured from the top of the valence band.

point X. Furthermore, the second lowest branch of the conduction band is slightly isolated from the continuous spectrum of the conduction band with higher energy.

To clarify the characteristics of the electronic structures of the nanowire, we have studied the detailed distribution of the squared wave function for the electronic states around the energy gap. Figures $5(\mathrm{~d})$, (e), and (f) show the wave function at the $\Gamma$ point at the top of the valence band, at the lowest branch of the conduction band, and at the second lowest branch of the conduction band, respectively. The valence band extends across the whole inner region of the wires exhibiting their covalent nature of $\mathrm{sp}^{3} \mathrm{C}$ atoms as in the case of bulk diamond, while the lowest branch of the conduction band is distributed along the edge of the wire exhibiting a one-dimensional character. More re- 
markably, the second lowest branch of the conduction band is distributed on the facet of the outermost shell with an anti-bonding $\pi$ character, indicating their two-dimensional character. Thus, the electronic structure of the diamond nanowires exhibits a dimensional interplay with the carrier injections for the extended and localized carrier distributions for the hole and electron doping, respectively. The characteristic distributions of the carriers are expected to be an advantage for the application of the nanowires as a conducting channel in nano-electronic devices.

\section{Summary}

We have studied the geometries, energetics, and electronic structures of diamond wire with nanometer scale diameter consisting of $\mathrm{sp}^{3} \mathrm{C}$ atoms. Graphitization of the outermost shell of the wire is found to take place by transformation from the $\mathrm{sp}^{3}$ to $\mathrm{sp}^{2}$ hybridizations of the surface atoms. Indeed, the optimized bond length in the outermost shell ranges from 0.134 to $0.149 \mathrm{~nm}$ indicating the evidence of $\mathrm{sp}^{2}$ rather than $\mathrm{sp}^{3}$ hybridization. In addition, significant elongation of the inter-shell bonds connecting the outermost and the inner shells is also found, indicating the possibility that the outermost shell can be peeled off from the core region of the diamond nanowire. The results indicate that the diamond nanowires could be a precursor for nanometer-scale materials consisting of $\mathrm{sp}^{2} \mathrm{C}$ atoms by successive peeling of their shells. Since the graphitization takes place in the outermost shell, the wires are energetically stable although their surfaces are not saturated by any chemical elements. The calculated total energy of the wire with $4.3 \mathrm{~nm}$ diameter is nearly equal to that of the single-walled nanotube with $1 \mathrm{~nm}$ diameter.

The graphitization also results in establishing the semiconducting electronic properties of the wires by decreasing the number of unpaired electrons associated with the dangling bonds. We find that the valence band extends across the whole inner region of the wires exhibiting their covalent nature of $\mathrm{sp}^{3}$ $\mathrm{C}$ atoms as in the case of bulk diamond. In sharp contrast, the electronic states in conduction band exhibits localized nature with the $\mathrm{sp}^{2}$ character: the lowest branch of the conduction band is distributed along the edge of the wire exhibiting a one-dimensional character, and the second lowest branch of the conduction band is distributed on the facet of the outermost shell with an anti-bonding $\pi$ character indicating their two-dimensional character. Thus, the electronic structure of the diamond nanowires exhibits a dimensional interplay with the carrier injections for the extended and localized carrier distributions for the hole and electron doping, respectively. The characteristic distributions of the carriers are expected to be an advantage for the application of the nanowires as a conducting channel in nano-electronic devices. 


\section{Acknowledgments}

We would like to thank Professor A. Oshiyama for providing the DFT program used in this work. This work was partly supported by CREST, the Japan Science and Technology Agency, and a Grant-in-Aid for scientific research from the Ministry of Education, Culture, Sports, Science and Technology of Japan. Computations were performed on an NEC SX-8/4B at the University of Tsukuba, on an NEC SX-8 at the Yukawa Institute of Theoretical Physics, Kyoto University, and on an NEC SX-9 at the Information Synergy Center, Tohoku University.

\section{References}

[1] S.J. Tans, A.R.M. Verschueren, C. Dekker, Nature, 393 (1998) 49.

[2] R. Martel, T. Schmidt, H.R. Shea, T. Hartel, Ph. Avouris, Appl. Phys. Lett. 73 (1998) 2447.

[3] K.S. Novoselov, A.K. Geim, S.V. Morozov, D. Jiang, Y. Zhang, S.V. Dubonos, I.V. Grigorieva, A.A. Firsov, Science 306 (2004) 666.

[4] S. Okada, S. Saito, A. Oshiyama, Phys. Rev. Lett. 83, 1986 (1999).

[5] N. Hamada, S.-I. Sawada, A. Oshiyama, Phys. Rev. Lett. 68, 1579 (1992).

[6] R. Saito, M. Fujita, M. S. Dresselhaus, G. Dresselhaus, Appl. Phys. Lett. 60 (1992) 2204.

[7] S. Saito, A. Oshiyama, Phys. Rev. Lett. 66 (1991) 2637.

[8] S. Okada, S. Saito, A. Oshiyama, Phys. Rev. Lett. 86 (2001) 3835.

[9] M. Fujita, K. Wakabayashi, K. Nakada, K. Kusakabe, J. Phys. Soc. Jpn. 65 (1996) 1920.

[10] K. Nakada, M. Fujita, G. Dresselhaus, M.S. Dresselhaus, Phys. Rev. B 54 (1996) 17954.

[11] S. Okada, A. Oshiyama, Phys. Rev. Lett. 87 (2001) 146803.

[12] S. Iijima, Nature, 354 (1991) 56.

[13] J.J. Boland, Adv. Phys. 42 (1993) 129.

[14] D. Haneman, Phys. Rev. 121 (1961) 1093.

[15] K.C. Pandey, Phys. Rev. Lett. 47 (1981) 1913.

[16] S. Okada, K. Shiraishi, A. Oshiyama, Phys. Rev. Lett. 90 (2003) 026803.

[17] T. Akiyama, K. Nakamura, T. Ito, Phys. Rev. B 74 (2006) 033307. 
[18] P. Hohenberg, W. Kohn, Phys. Rev. 136 (1964) B864.

[19] W. Kohn, L.J. Sham, Phys. Rev. 140 (1965) A1133.

[20] J.P. Perdew, A. Zunger, Phys. Rev. B 23 (1981) 5048.

[21] D.M. Ceperley, B. J. Alder, Phys. Rev. Lett. 45 (1980) 566.

[22] L. Kleinman, D.M. Bylander, Phys. Rev. Lett. 48, (1982) 1425.

[23] Y. Miyamoto, A. Rubio, X. Blase, M.L. Cohen, S.G. Louie, Phys. Rev. Lett. 74 (1995) 2993.

[24] J.-Y. Raty, G. Galli, C. Bostedt, T.W. van Buuren, L.J. Terminello, Phys. Rev. Lett. 90 (2003) 037401.

[25] D. Takagi, Y. Kobayashi, H. Hibino, S. Suzuki, Y. Homma, Nano Lett. 8 (2008) 832. 